\title{
Les tilleuls du ministère de la Guerre
}

\section{Karin Gundersen}

\section{OpenEdition}

Journals

Édition électronique

URL : http://journals.openedition.org/recherchestravaux/350

DOI : 10.4000/recherchestravaux.350

ISSN : 1969-6434

\section{Éditeur}

UGA Éditions/Université Grenoble Alpes

\section{Édition imprimée}

Date de publication : 15 juillet 2009

Pagination : 69-77

ISBN : 978-2-84310-146-5

ISSN : 0151-1874

\section{Référence électronique}

Karin Gundersen, «Les tilleuls du ministère de la Guerre », Recherches \& Travaux [En ligne], 74 | 2009, mis en ligne le 28 février 2011, consulté le 08 septembre 2020. URL : http://journals.openedition.org/ recherchestravaux/350; DOI : https://doi.org/10.4000/recherchestravaux.350 
Karin GUNDERSEN

Université d'Oslo

\section{Les tilleuls du ministère de la Guerre}

En mai 2006 ma traduction de la Vie de Henry Brulard: Henry Brulards liv a paru aux Éditions Gyldendal à Oslor. L'événement a été bien accueilli et commenté par la presse norvégienne, où les critiques littéraires ont loué entre autres choses l'étonnante actualité de Stendhal, comparant Brulard aux textes tout récents de Winfried Georg Sebald et Thomas Bernhard². D'où vient cette impression de fraîcheur quand on lit certains textes de Stendhal? C'est la question à laquelle je vais tâcher de répondre, c'est-à-dire d'apporter une réponse parmi d'autres, celle d'une lectrice-traductrice. Dans ce contexte (celui de la fraîcheur) je donne aux tilleuls - qui surgissent par-ci par-là dans l'œuvre stendhalienne - une valeur emblématique qui revient aux arbres comme tels et s'exprime avec une clarté éminente dans les tilleuls situés au début du chapitre 4I de Brulard, rafraîchissant de leur mieux le jardin du ministère de la Guerre, à Paris, vers la fin de l'hiver I 800 . Henry a dix-sept ans et travaille au ministère, ne sachant pas encore que très bientôt il va traverser les Alpes avec l'armée de réserve de Napoléon. Stendhal par contre est en Italie depuis longtemps quand, trente-six ans plus tard, il se souvient des tilleuls de Paris en écrivant la page que je vais commenter.

La traduction «rend manifestes les structures cachées d'un texte»; mieux : elle «potentialise» le texte, dit Antoine Berman en se référant à Ezra Pound et Novalis 3 . Encore faudra-t-il que le texte soit en forme, pour ainsi dire. Or la preuve ultime qu'un texte classique se porte bien et conserve toute sa vigueur, c'est qu'il est amusant à traduire. La traduction est une activité

I. J'avais déjà traduit La Chartreuse de Parme (1992), De l'amour (1995), et Le Rouge et le Noir (200I), tous chez le même éditeur.

2. La réception de Henry Brulards liv en Norvège est récapitulée et commentée par J. Holm dans L'Année stendhalienne, $\mathrm{n}^{\circ}$ 6, 2007, p. 393-395.

3. L'Éprenve de l'étranger, Gallimard, «Tel», I984, p. 20. 
solitaire et silencieuse. Elle est solitaire au sens de triste et morne quand le texte est mort, et j'en ai traduit, des textes plus ou moins morts, même modernes. Alors on s'ennuie. Quand on a le bonheur de traduire un texte vivant et bien portant, on est seul aussi. Mais on ne s'ennuie pas. C'est difficile d'expliquer ce qui se passe, et qui fait que je ne sacrifierais pas une soirée et une nuit avec Stendhal pour n'importe qui. Voici une amorce d'explication de l'expérience singulière.

La traduction se fait en silence. Le traducteur reste longtemps pensif, entre les phrases, entre les mots, dans un espace intermédiaire où l'on voit se rapprocher, comme dans une espèce de danse, les corps des deux langues. On contemple leurs affinités et contradictions, on s'étonne de la générosité de l'une qui répond à l'insuffisance de l'autre. À tour de rôle. Le traducteur voit les mots venir, et parfois hélas, il les voit disparaitre : s'éloigner au ralenti comme une chose rêvée au moment du réveil. La pulsion du traduire ${ }^{4}$ a ses déceptions et ses succès comme toute autre pulsion; disons que le traducteur se sent ultra-satisfait quand il arrive au stade dont parle Walter Benjamin, où son rapport au texte se double d'une vision du langage pur.

Ainsi la traduction, encore qu'elle ne puisse élever une prétention à la durée de ses ouvrages, $[\ldots]$ ne renonce pas pour autant à s'orienter vers un stade ultime, définitif et décisif, de tout assemblage langagier. En elle l'original croît et s'élève dans une atmosphère, pour ainsi dire, plus haute et plus pure, du langage, $[\ldots]$ indiquant le royaume promis et interdit où les langues se réconcilieront et s'accompliront. Ce royaume, il ne l'atteint jamais complètement, mais c'est là que se trouve ce qui fait que traduire est plus que communiquer. ${ }^{5}$.

Traduire est plus que communiquer. Cette vérité nous ramène rapidement au niveau de la pratique. La traduction est en pratique une lecture et une interprétation. Pour interpréter il faut écouter. Le traducteur est à l'écoute du texte qui nous parle dans ses plus petits détails. Quand je traduis, je ne peux pas sauter les parties ennuyeuses ou difficiles, ou lire ce que je crois reconnaitre parce que je l'ai lu ailleurs ou déjà compris par l'effet circulaire de l'herméneutique 6 . Comme traductrice, je dois regarder chaque phrase et chaque mot comme un témoin qui m'appelle. Comme la trace d'un monde sensible, d'une expérience ou d'un souvenir qui demande à être reprise, retracée et rappelée à la vie. Cet effet de trace, qu'il ne faut pas confondre avec l'aspect physique de l'écriture,

4. Cette belle expression est de Berman, op. cit., p. 2 I.

5. «La tâche du traducteur», dans CEuvres, t. I, Mythe et violence, trad. M. de Gandillac, Denoël, I971, p. 267-268.

6. Par exemple, si Fabrice del Dongo est beau et que dans les romans, la beauté a tendance à être corrélative à la bonté, je puis en tant que lecteur fermer les yeux à la méchanceté de «notre héros». Que Stendhal l'ait marquée comme telle, par des subtils détails, c’est ce que je ne peux pas éviter de voir en traduisant. 
figure le geste qui porte le texte et le fait passer d'un bord à l'autre. Car c'est en se ressouvenant lui-même, en remontant ses propres sentiers, que le traducteur revient au monde des vivants avec une sensation toute fraîche et une pensée nouvelle. Voilà pourquoi la traduction est une valeur intrinsèque. En supplément à son utilité pour le lecteur qui ne comprend pas la langue de l'original, et à son rôle essentiel pour l'œuvre qu'elle fait revivre dans toute sa splendeur selon Benjamin, elle est une valeur pour le traducteur lui-même qui, dans la solitude et le silence de son vis-à-vis avec le texte, «s'amuse», se passionne, désespère ou triomphe. Rappelons qu'au niveau de l'art la traduction peut être regardée comme la matrice de l'écriture, selon le dit célèbre de Proust dans Le Temps retrouvé:

[...] je m'apercevais que le livre essentiel, le seul livre vrai, un grand écrivain n'a pas, dans le sens courant, à l'inventer, puisqu'il existe déjà en chacun de nous, mais à le traduire. Le devoir et la tâche d'un écrivain sont ceux d'un traducteur?.

Pour faire revivre un texte il ne suffit pas de traduire les phrases et les mots, il faut aussi et d'une manière encore plus attentive écouter la ponctuation, les italiques, les blancs, les silences et le ton. En I984, dans un article de la revue suédoise Kris, Horace Engdahl parle du style de Stendhal par rapport au $\operatorname{ton}^{8}$. Au niveau le plus immédiat, le «ton», dit Engdahl, c'est ce qui marque l'attitude du sujet énonciateur envers son propre énoncé et sa part d'engagement dans la matière concernée. Il cite Derrida disant que le ton, c'est le geste du mot, irréductible à l'analyse linguistique, sémantique ou rhétorique de l'énoncé'; ensuite il se réfère à Blanchot selon qui le ton, c'est la vibration du temps réel dans l'intemporel de l'écrit, le silence imposé à la parole :

Écrire, c'est se faire l'écho de ce qui ne peut cesser de parler - et, à cause de cela, pour en devenir l'écho, je dois d'une certaine manière lui imposer silence. J'apporte à cette parole incessante la décision, l'autorité de mon silence propre. [...] Ce silence a sa source dans l'effacement auquel celui qui écrit est invité. Ou bien, il est la ressource de sa maitrise, ce droit d'intervenir que garde la main qui n'écrit pas, la part de lui-même qui peut toujours dire non et, quand il le faut, en appelle au temps, restaure l'avenir.

Lorsque, dans une œuvre, nous en admirons le ton, sensibles au ton comme à ce qu'elle a de plus authentique, que désignons-nous par là? Non pas le style, ni l'intérêt et la qualité du langage, mais précisément ce silence, cette force virile [...]

Le ton n'est pas la voix de l'écrivain, mais l'intimité du silence qu'il impose à la parole, ce qui fait que ce silence est encore le sien, ce qui reste de lui-même dans

7. À la recherche du temps perdu [1954], t. III, P. Clarac et A. Ferré éd., Gallimard, «Bibliothèque de la Pléiade», I 977 , p. 890.

8. «Stilen och lyckan» (Le style et le bonheur), Kris, nos 29-30, novembre i 984, p. 5-I 2.

9. Les fins de l'homme. À partir du travail de Jacques Derrida, Colloque de Cerisy sous la direction de Ph. Lacoue-Labarthe et J.-L. Nancy, Galilée, I98 I, p. 477. Rappelons le titre de la contribution de Derrida : «D’un ton apocalyptique adopté naguère en philosophie». 
la discrétion qui le met à l'écart. Le ton fait les grands écrivains, mais peut-être l'œuvre ne se soucie-t-elle pas de ce qui les fait grands ${ }^{10}$.

Pour Engdahl, le ton est un aspect fondamental de l'écriture de Stendhal dans la mesure où ce sont le ou plutôt les tons et leurs variations de phrase en phrase qui font naitre son style. Tandis que l'on reconnait le style personnel d'autres grands écrivains aux métaphores, descriptions, lieux et lumières qui leur sont propres, le «monde sensible» de Stendhal se présente à nous d'une façon assez fruste et frugale. En revanche, il y a chez Stendhal ce que l'on pourrait selon Engdahl appeler une chorégraphie mentale, très sensible dans ses écrits autobiographiques, qui consiste en un changement de perspective, de vitesse et d'appréciation qui se déploie à partir du rapport entre deux lieux temporels séparés par des années voire des décennies. Cela crée une dimension temporelle intérieure et intime, celle du vécu, mais qui chez Stendhal devient réversible. Le retour du souvenir, au lieu de se figer en image, fonctionne plutôt comme déclencheur et transforme «Stendhal» écrivain en une frontière mouvante vers le déjà-écrit.

Dans son article " "Approche" stendhalienne», Jacques Neefs ${ }^{11}$ recoupe la même problématique, celle du silence quand l'écriture est interrompue dans sa course pour apprécier un souvenir qui réapparaît en sa présence immédiate. Réfléchissant sur la vitesse de l'écriture de Stendhal et son effort pour rattraper l'afflux des souvenirs et s'approcher du vécu - mouvement dans lequel il emporte le lecteur - Jacques Neefs va droit au cœur de la chose qui me concerne, dans son commentaire d'un certain passage de Brulard (celui de la pêche à la truite avec Monsieur de Corbeau, au chapitre I3, "Premier voyage aux Échelles») :

L'afflux des souvenirs sur la page brouille l'écriture qui tente de saisir ceux-ci. [...] Mais l'écriture s'est précisément interrompue, dans son crépuscule, avec le surgissement d'un souvenir de bonheur, quand l'assomption d'une joie enfantine a été retrouvée :

«Vers Chailles, en relevant vivement l'hameçon, sa [...] ligne de crin blanc passa sur un arbre, et la truite de trois quarts de livre (ce sont les bonnes) nous apparut pendant à vingt pieds de terre au haut de l'arbre qui était sans feuilles. Quelle joie pour moi!»

La vision baroque d'un poisson dans les arbres surgit comme un rire, le monde un instant à l'envers flotte dans la mémoire, et l'écriture coïncide avec l'enfant : «Quelle joie pour moi ${ }^{12} ! »$

ı. M. Blanchot, «La solitude essentielle», L'Espace littéraire [1955], Gallimard, «Folio Essais», I 993, p. 2 I-22.

I I. "“Approche" stendhalienne», Figura. Festskrift for Karin Gundersen, G. Bjørnerud M. et J. Holm éd., Oslo, Unipub, 2004, p. I94-I 99.

I 2. Ibid., p. 195. 
Dans ce fragment de texte merveilleux, il y a aussi les parenthèses qui abritent la note de l'expert, consul de France à Civitavecchia, ancien haut fonctionnaire de l'Empire, homme à s'y connaître en matière de bonne chère : les truites de trois quarts de livre, ce sont les bonnes! L'écrivain surgit devant nous à sa table de travail, juste avant l'heure du dîner, songeant à la bonne truite.

Attitude, geste ou silence, le ton ne serait pas à repérer directement dans la phrase ou le mot comme tels, mais entre et à travers eux. Entre les phrases stendhaliennes il se passe parfois beaucoup, tout à fait comme dans la traduction : là, il y a parfaite coïncidence. Voilà peut-être une des raisons pour lesquelles Stendhal est tellement passionnant à traduire. Il y a coïncidence ou accord, comme une espèce de musique entamée et reprise de phrase en phrase. Le défi de la traduction et la responsabilité du traducteur en augmentent proportionnellement : comment transmettre ce qui se passe entre les phrases? Et quelles phrases! Comment faire pour ne pas écraser, dans le transfert à l'étranger, cette musique silencieuse, le ton?

Le ton est inséparable de ce qui est dit. Cela semble aller de soi; or, ce que Stendhal dit vise toujours «le fond des choses». Ainsi entend-il prendre ses distances avec Jean-Jacques Rousseau, charlatan, qui s'est appliqué à cacher son imposture dans un beau style ${ }^{13}$.

On n'a pas besoin de «style» quand on est éclairé par le réel, par l'idée véridique d'une chose réelle. Dans son article «Stendhal entre les phrases», Stéphane Chaudier rappelle que «chez Stendhal, il y a plus de pensées que de mots $^{14}{ } \gg$. Ce phénomène, Chaudier l'analyse à l'aide d'une structure logique tripartite retrouvée chez Stendhal : «fait, conséquence, commentaire», qui montre à la fois la force du réel et le rythme de la déterritorialisation qui s'opère dans le texte; autrement dit : la volonté de ne pas rester près du déjà acquis. Il faut aller plus loin et ailleurs. D'où la jubilation des vérités qui se succèdent, qu'elles soient tristes ou plaisantes. La satisfaction intellectuelle de capter une vérité rachète sa douleur éventuelle.

J'arrive au texte, à mes tilleuls. Nous allons lire le début du chapitre 4I de la Vie de Henry Brulard, que je vais couper en séquences pour faciliter le dialogue avec le texte :

Au bout du jardin étaient de malheureux tilleuls taillés de près derrière lesquels nous allions pisser. Ce furent les premiers amis que j'eus à Paris. Leur sort me fit pitié : être ainsi taillés! je les comparais aux beaux tilleuls de Claix qui avaient le

I3. Paraphrasé d'après Engdahl (art. cité), qui se réfère aux Mélanges intimes et marginalia, vol. 2, H. Martineau éd., Le Divan, i 936, p. 287.

I4. "Stendhal entre les phrases», Stendhal et le style, Ph. Berthier et É. Bordas éd., Presses Sorbonne Nouvelle, 2005, p. I44. 
bonheur de vivre au milieu des montagnes.

Mais aurais-je voulu retourner dans ces montagnes ${ }^{15}$ ?

Le thème du malheur, projeté sur les arbres anthropomorphisés, est doublé par la présence du corps ${ }^{16}$, de la «machine», dirait Stendhal, qui malgré cela est indispensable à la sensibilité, à l'émotion et à la tendresse. Et à la compassion : «Leur sort me fit pitié [...] je les comparais aux beaux tilleuls de Claix [...]» À l'indignation aussi (avec point d'exclamation, espèce de deixis qui marque la présence de l'écrivain) : «être ainsi taillés!» Dans De l'Amour, Stendhal comparait les femmes aux arbres du jardin de Versailles; comme eux, elles sont «taillées». Mais peut-on en conclure qu'elles naissent ainsi ${ }^{17}$ ? Pauvres arbres, pauvres femmes! Il y a pourtant une région où les arbres sont heureux, à Claix, près de Grenoble, patrie haïe! La contradiction du double lieu : celui du bonheur (pour mes amis tilleuls de Claix) qui est en même temps le lieu du malheur (pour moi), cette idée silencieuse marquée par l'alinéa, provoque à son tour la question suivante, dite d'un ton incrédule : «Mais aurais-je voulu retourner dans ces montagnes?» Réponse :

Oui, ce me semble, si j'avais dû n'y pas retrouver mon père et y vivre avec mon grand-père, à la bonne heure, mais libre.

Voilà à quel point mon extrême passion pour Paris était tombée. Et il m’arrivait de dire que le véritable Paris était invisible à mes yeux.

Entre ces deux paragraphes il y a toute l'enfance malheureuse d'Henry. La liberté en italique formule le désir répété de l'enfant qui se sentait enfermé dans la maison de son père, petit enfant solitaire, sans amis (sauf un vieillard: son grand-père). Une solitude pareille domine son premier séjour à Paris, cette ville dont il a tellement rêvé, mais qui lui est hostile jusqu'au point de le faire rêver de «retourner dans [ses] montagnes». À Paris, ses premiers voire seuls amis sont quelques «malheureux tilleuls». Pour eux, l'amitié d'Henry est quand même un petit bonheur, tout taillés et misérables qu'ils sont. Car voici ce qui leur arrive, un jour :

Les tilleuls du ministère de la Guerre rougirent par le haut. M. Mazö̈er sans doute me rappela le vers de Virgile :

\section{Nunc erubescit ver}

Ce n'est pas cela mais je me le rappelle, en écrivant, pour la première fois depuis trente-six ans; Virgile me faisait horreur au fond, comme protégé par les prêtres

I 5. P. 386-387 dans l'édition de B. Didier [1973], Gallimard, «Folio», I988.

I6. Grâce à l'occurrence du verbe "pisser», rare dans les belles-lettres. On ne pisse guère dans la littérature entre Rabelais et Joyce. Même plus tard, chez Beckett, on le fait en périphrases.

17. Chap. LIV p. 208 dans l'édition de V. Del Litto [1980], Gallimard, «Folio», I 998 . Il y a tout un réseau d'arbres dans l'œuvre stendhalienne qu'il faudrait un jour explorer. 
qui venaient dire la messe et me parler de latin chez mes parents. Jamais, malgré tous les efforts de ma raison, Virgile ne s'est relevé pour moi des effets de cette mauvaise compagnie.

À la mauvaise compagnie des prêtres (sales et mal élevés en plus! le lecteur s'en souvient bien) - les tilleuls font contraste par leur émouvante rougeur involontaire, parole du corps, comme chez les jeunes filles timides qui témoignent de leur tendresse en rougissant jusqu'aux yeux... Cette image, que les uns diraient se trouver uniquement dans la tête de la traductrice, reprend pourtant l'allégorique arbre-jeune femme qui, au moins depuis l'histoire d'Apollon et Daphné, est un topos de la littérature et la poésie de l'amour ${ }^{18}$.

Nous sommes déjà passés, en peu de mots, de Paris à Grenoble en compagnie du jeune Henry; nous voici à Rome, la Rome ancienne invoquée au début de Brulard, celle de Virgile, Ovide et les autres. Malgré le fait que ce vers soit apocryphe - on ne l'a pas retrouvé chez Virgile - son expression ainsi que son thème («Maintenant rougit le printemps») sont du bon latin. Toute la culture classique se présente à nous, pour faire résonner le souvenir personnel de Stendhal, sa vie intime, dans notre histoire à tous.

Arbres qui rougissent par le haut : c'est d'abord un signe de printemps (les premières pousses rougeâtres des feuilles), qui pourra ensuite se lire comme un effet de lumière, et notamment d'un coucher de soleil, comme une lueur du jour qui reste sur le haut des branches. Lueur qui figure le ton propre du souvenir, analogue en cela à la littérature qui, au dire de Roland Barthes, est la lueur même du réel :

$\mathrm{Si}$, par je ne sais quel excès de socialisme ou de barbarie, toutes nos disciplines devaient être expulsées de l'enseignement sauf une, c'est la discipline littéraire qui devrait être sauvée, car toutes les sciences sont présentes dans le monument littéraire. C'est en cela que l'on peut dire que la littérature, quelles que soient les écoles au nom desquelles elle se déclare, est absolument, catégoriquement, réaliste : elle est la réalité, c'est-à-dire la lueur même du réel ${ }^{19}$.

Mais revenons à nos arbres. Pendant que nous étions dans l'ancienne Rome, ayant étendu l'espace de la mémoire (trente-six ans dans le texte) jusqu'à deux millénaires ou presque, il s'est passé ceci :

Les tilleuls prirent des bourgeons, enfin ils eurent des feuilles, je fus profondément attendri, j'avais donc des amis à Paris!

Chaque fois que j'allais pisser derrière ces tilleuls au bout du jardin, mon âme était rafraîchie par la vue de ces amis. Je les aime encore après trente-six ans de séparation.

I 8. Je me borne à rappeler la rose/le rosier de Ronsard : «Mignonne, allons voir...»

I 9. R. Barthes, Leçon, Seuil, I 978 , p. I 8. 
Le texte sourit : prirent, attendri, amis; pisser, tilleuls, rafraîchie, amis, trentesix. À ce souvenir, l'âme est rafraîchie et tout le monde sourit, y compris la traductrice qui, par bonheur, a trouvé le participe passé, oppfrisket en norvégien, qui avec son $i$ dominant sonne comme un sourire aussi, et elle l'a mis en italique bien sûr. Pour souligner le ton souriant du passage, au souvenir de l'amitié : «Je les aime encore après trente-six ans de séparation.» Pause, silence de la mélancolie douce : être ainsi séparé de ses amis (souvenir parallèle du lecteur/de la traductrice), peut-être pour toujours :

Mais ces bons amis existent-ils? On a tant bâti dans ce quartier! Peut-être le ministère où je pris la plume officielle pour la première fois est-il encore le ministère rue de l'Université, vis-à-vis la place dont j'ignore le nom?

Le ton de la séparation et du deuil (rappel du bonheur perdu : arbres amis représentants de tous les amis disparus) que la "plume» intime véhicule et où l'on risque de s'attendrir sur soi-même, sera heureusement coupé par «la plume officielle». Du sourire attendri qui nous amène au bord du sentimentalisme, dépêchons-nous de revenir. Revenons à la sécheresse, voire à la dérision, et rions de nous-mêmes :

Là M. Daru m'établit à un bureau et me dit de copier une lettre. Je ne dirai rien de mon écriture en pieds de mouche, bien pire que la présente, mais il découvrit que j'écrivais cela par deux 11 : cella.

C'était donc là ce littérateur, ce brillant bumaniste qui discutait le mérite de Racine et qui avait remporté tous les prix de Grenoble!

Le sarcasme du «brillant humaniste», même lu comme discours indirect libre (l'italique bumaniste pourrait indiquer une citation), chasse le sentimentalisme qui guettait, et aussi la vanité peut-être, car l'écrivain n'a-t-il pas failli avoir la présomption de se comparer à Virgile?

Dans son «Stendhal et les âmes sensibles ${ }^{20} »$, Blanchot dit que «le trait le plus propre à éclairer le charme particulier de Stendhal [est] une certaine sensibilité de l'âme ${ }^{21} »$. Il le rapproche du «naturel» proprement stendhalien, de sa haine de l'ennui et son goût de la spontanéité. Et il continue :

Si l'on accepte, par jeu, de chercher dans sa correspondance quel est le caractère de ce naturel insaisissable, l'on sera facilement tenté de le réduire à un certain ton, à une manière d'écrire opposée au développement et au style soutenu, à une vivacité d'expression qui imite la conversation dans ce qu'elle a d'immédiat et s'en écarte par un tour plus individuel et plus spécifique ${ }^{22}$ ?

C'est justement ce ton de la conversation que nous venons d'identifier dans ce qui précède : la parole de Stendhal avec ses coupures, ses pauses, silences

20. Compte rendu d'un recueil (édité par Boudot-Lamotte) de lettres de Stendhal.

21. M. Blanchot, Faux Pas [1 943], Gallimard, I971, p. 301.

22. Ibid., p. 30I-302. 
et rapides changements de vitesse, avec tout ce qui fait qu'il y a "plus de pensées que de mots $^{23}$ ». Cela, combiné avec la sécheresse de l'expression, nous donne ce que nous aimons, ce grâce à quoi nous ne nous ennuyons jamais en sa compagnie, absence d'ennui, présence du bonheur. Dans la prose de Blanchot :

La sécheresse même de l'expression stendhalienne n'est en réalité que le chemin le plus court pour atteindre à une extrême richesse d'émotion et les notations de son «algébrisme» sont un appel brusque et bref, destiné à réveiller le plus cher souvenir, à réintroduire au milieu des circonstances misérables ou médiocres les vives surprises du cœur, telles qu'elles lui ont été connues une fois dans leur puretée ${ }^{24}$

Il y a une éthique de ce ton : l'éthique de la fraîcheur, celle qui n'ennuie jamais, qui tient en éveil tout traducteur, sinon tout lecteur, la nuit entière. Dans un texte qu'on a tendance à oublier, mais qui m'appelle maintenant, Barthes dit :

Or toute Forme est aussi une Valeur; c'est pourquoi entre la langue et le style, il y a place pour une autre réalité : l'écriture. Dans n'importe quelle forme littéraire, il y a le choix général d'un ton, d'un éthos, si l'on veut, et c'est ici précisément que l'écrivain s'individualise clairement parce que c'est ici qu'il s'engage ${ }^{25}$.

Si près de nous, malgré les deux siècles qui nous séparent, Stendhal se soucie de la santé de notre âme et rétablit une fraîcheur du langage ${ }^{26}$ qui nous permet de respirer.

23. Voir ci-dessus, la citation de S. Chaudier.

24. Op. cit., p. 305.

25. Le Degré zéro de l'écriture [1953], Seuil, «Points», I 972, p. I4.

26. Encore qu'elle ne soit pas destinée à Stendhal de façon explicite, cette belle phrase de Barthes mérite d'être citée : «[l'écriture littéraire] se hâte vers un langage rêvé dont la fraîcheur, par une sorte d'anticipation idéale, figurerait la perfection d'un nouveau monde adamique où le langage ne serait plus aliéné» (ibid., p. 64-65). 